\title{
The Investigation on Redundancy Errors in Writing of Chinese English Learners with Different Proficiency
}

\author{
Yujing Yang ${ }^{1, *}$ \\ ${ }^{1}$ School of International Studies, Wenzhou Business College, 325035, Wenzhou, Zhejiang, China \\ *Corresponding author. Email: yangyujing@wzbc.edu.cn*
}

\begin{abstract}
This research paper aims to figure out the main categories of redundancy errors in English writing tasks for Chinese undergraduates as well as the relations between different kinds of redundant constituents and learners' English proficiency. Taking error analysis as the theoretical framework, this research aims to conduct a corpus-based study firstly and written discourse analysis accordingly. To find the distribution of different redundancy errors under the setting of English writing tasks, this research selects the Chinese Learner English Corpus (abbr. CLEC) as the source to search and classify redundancy errors. Sixty non-English majors with different English proficiency from a university in China are involved in the study. They are assigned a questionnaire consisting of fourteen sentences to judge whether there are redundancy errors, and then their answers are used for further data analysis. It is thought in this paper that the reasons for redundancy errors consist of language transfer and inadequate knowledge of the target language. In addition, this research puts forwards some practical strategies to be adopted for Chinese students to improve their English writing skills. This paper regards that students should avoid message repetition or entailment by realizing the semantic overlap between words. It is also referred students should raise the awareness of strengthening the grammar rules, thus preventing the overuse of functional words.
\end{abstract}

Keywords: Redundancy Errors, Repetition, Message Entailment, Error Analysis, Language Proficiency, Negative Transfer, Interlanguage.

\section{INTRODUCTION}

In message transmission, people tend to send more information than needed to meet the minimum amount of understanding [1]. The part of the information that is more than the minimum required is redundant. Redundancy is a common phenomenon in language, both in the language system itself and in everyday communication. However, unnecessary redundancy neither provides more new information nor has any rhetorical or literary function, which greatly affects the accuracy and readability of the written content [2]. This research paper intends to investigate the main categories of redundancy errors and their relationships with language proficiency for non-English major students in China under the setting of English writing tasks from the perspectives of semantics and syntax. Under the framework of error analysis, the paper adopts the research methods of corpus-based analysis and annotation test to analyze students' most common and frequent redundancy errors by analyzing results from productive and receptive tasks step by step. The research questions are as follows:

(1) What redundancy errors would L2 learners make in writing tasks?

(2) Will L2 learners' English proficiency influence their common redundancy error types?

\section{LITERATURE REVIEW}

\subsection{The definition of redundancy}

Originally introduced by Shannon, redundancy is a concept in information theory that refers to extra information which is released as a supplement for the core information to effectively reduce interference in information transmission [1]. According to Rosie, anything other than the minimum required to represent or transmit information is considered redundant [3]. Reza also argues that since information is always disturbed by noise when it is transmitted in the channel, the information sender intends to encode more 
information than the actual need to eliminate the negative impact of information loss [4]. Overall, these scholars outline a crucial role of redundancy and define it as excessive information provided in information transmission beyond a minimum amount to ensure the effectiveness of communication. Extending this concept into linguistics, $\mathrm{Xu}$ points out that the coexistence of extra information with an essential message in a language is seen as linguistic redundancy which is mainly embodied in message repetition and message entailment [5]. The former is expressed by the repetition of the same language units or the reoccurrence of expressions of homologous and synonymous relation while the latter means that the information carried by one language unit is contained by another one.

\subsection{The classification of redundancy}

A preliminary consensus on the classification for redundancy has been achieved among researchers. Classifying it into linguistic redundancy and non-linguistic redundancy, researchers assume a dichotomy based on the causes for emergence whether the redundancy is generated from grammatical rules or the contextual need.

According to Gillette and Wit, "Grammatical redundancy is the internal systematicity and rule-governed behavior of a language in which two or more its features serve the same function. Grammatical redundancy is obligatory" [6]. Xu also illustrates the function of this category of redundancy by declaring that they serve as grammatical markers but do not convey lexical meaning [5]. Shao enlarges the scope of the grammatical redundancy by giving examples of tautological expressions as examples of the quadrisyllabic in Chinese which is also inherent in some particular language systems [7].

Non-linguistic redundancy or contextual redundancy is applied between interlocutors for socio-linguistic and psycho-linguistic factors. Words or expressions are repeated intentionally to facilitate communication or achieve a certain rhetorical effect [6]. In this sense, Wang refines semantic repetition by dividing them into necessary redundancy and unnecessary redundancy [2]. Only the "semantic copying" that enhances the language effect can be regarded as necessary, otherwise, they are unnecessary synonymous repetitions [2].

Another classification comes from Liu and Xiang by dividing redundancy into semantic redundancy and structural redundancy [8]. Semantic redundancy refers to the repetitions of meaning from words or phrases, which is mainly caused by the repetition or entailment of content words. Structural redundancy mainly results from the overuse of functional words, such as prepositions, articles.
Through integrating the classification in previous research, redundancy errors in this paper are classified into semantic redundancy errors and syntactic redundancy errors, which are based on the classification of Liu and Xiang [8].

\subsection{Previous research on redundancy errors}

Notably, the corpus-based approach gains the most popularity since many researchers conducted discourse analysis to find the distribution of redundancy errors. Redundancy errors are also usually investigated together with omission errors. $\mathrm{Li}$ and Cai focus on the omission and redundancy errors of articles committed by Chinese learners through analyzing the writing samples from CLEC [9]. The results from their calculations show redundant articles are mostly intralingual errors. Liu and Liu also select writing samples from CLEC for data statistics [10]. They calculate the frequency of redundancy errors as subdivisions of articles, propositions, conjunctions and pronouns out of 10,000 words and rank the statistical results, finding the top three subdivisions of redundancy errors are definite articles, simple prepositions and conjunctions. In addition, some frequently used conjunctions including "and", "but" and "so" are studied by an increasing number of researchers [11][12][13]. Jiang conducts a comprehensive survey based on CLEC and concludes that redundant constituents concentrate in five parts of speech, constituting nearly $80 \%$ among lexical redundancy errors [14]. Arranged in descending order, they are prepositions, articles, nouns, conjunctions and adverbs in turn. Cai conducts case studies by analyzing redundancy errors in three linguistic levels including sentence, paragraph and discourse [15]. She tries to explain the redundancy errors based on negative transfer from philosophic thoughts, pragmatics and habitual way of Chinese.

Overall, although many researchers have studied Chinese college students' redundancy errors under the theoretical model of error analysis, two main research gaps still exist for future study. Firstly, since the previous corpus-based studies focus on particular kinds of redundancy errors, nearly no comprehensive demonstration of the distribution of redundancy errors from each part of speech has been shown from the corpus because of the huge workload. Secondly, the researchers can only summarize the categories and subsets of redundancy errors committed by some L2 learners but do not know whether they are universal for all EFL learners in China because of the potential for avoidance [16]

\section{METHODOLOGY}

Both quantitative and qualitative research approaches are used in this study. The quantitative 
approach is utilized in investigating the distribution and categories redundancy errors from the writing samples in corpus and comparing the different results made by the students with different language proficiency. The qualitative approach is employed in analyzing the causes of redundancy errors.

\subsection{Corpus-based study}

This research has chosen the Chinese Learner English Corpus (abbr. CLEC) as the source which collects a total of more than one million words of written samples from five types of students, including secondary school students, non-English majors (CET-4), non-English majors (CET-6), freshmen and sophomores in English major, and juniors and seniors in English major. All the errors in CLEC have been marked by compliers who give each type of errors a tag in brackets.

In the corpus-based study, redundancy errors with a marker [wd-5] are extracted by concordance tool AntConc from the college students' writing samples in CLEC. The redundancy errors are classified into two main categories and ten subsets.

\subsection{Annotation}

The participants involved in the present study are 60 undergraduates in a university in southern China, whose ages range from 18-20 years old. All of them are non-English majors.. 30 of them are in Grade 1 and Grade 2 who have passed CET- 4 while another 30 are in Grade 3 and 4 who have passed CET-6. They are described in this paper as students of the upper-level group and lower-level group.

The annotation test chooses fourteen sentences from the previous step, each containing one subset of redundancy error as the test material. Seven of them contain a redundant content word or phrase while the other seven contain a redundant functional word. The questionnaires are assigned to students to judge whether the sentences contain redundant constituents. After collecting data from two groups, a comparative analysis is conducted to reveal students' recognition towards different categories and subsets of redundancy errors at different language proficiency. The results allow the researcher to find out which categories or subsets of redundancy errors are common among Chinese English learners.

\section{RESULTS}

\subsection{Distribution of redundancy errors}

Data are collected from four subsets from st3 to st6 as the collections of college students' writing samples from CLEC, and redundancy errors of each part of speech within sentences are collected to offer a comprehensive collection and classification of redundancy errors. Constituents with semantic repetition or entailment are underlined while syntactic redundancy errors are labeled with wavy lines in the following examples for a clearer demonstration.

\section{Example 1 Noun Redundancy}

"I should like to apply for a secretary job."

The noun "job" is normally used with the verb phrase "apply for" and placed after the phrase. Another noun "secretary" in front of "job" is a redundant modifier since it is a particular type of job.

\section{Example 2 Pronoun Redundancy}

"Students have to pay their tuition fees themselves."

Both the possessive pronoun "their" and the reflexive pronoun "themselves" noun "job" refer to the same subject. One of them should be removed since there is message repetition.

\section{Example 3 Verb Redundancy}

"There are other interesting and special means of transportation remained in China."

In this existential sentence, "are" has already implied the meaning that sth. remains in somewhere. The verb "remain" also describing the geographic position of transportation is a redundant constituent in this sentence]

\section{Example 4 Adjective Redundancy}

"There are all kinds of different Zongzi all over the country."

The two modifiers share the same meaning that Zongzi, the traditional Chinese rice pudding, displays a variety. There is no need to apply the adjective phrase "all kinds of" and the adjective "different" to modify the same noun "Zongzi".

\section{Example 5 Adverb Redundancy}

"A lot of people compete together for one job, of course some of them will lose."

The verb "compete" implies the involvement of more than one subject, so the meaning of the adverb "together" is already implied in the word it modifies.

\section{Example 6 Adverb Redundancy}

\section{"In China, our situation is relatively better."}

The modifier "relatively" means "by comparison to something else". However, "better", as a comparative adjective, also contains the meaning of a comparative state. Message entailment is contained in this adverb-adjective collocation.

$\begin{array}{cccc}\text { Example } & 7 & \text { Coordinating } & \text { Conjunction } \\ \text { Redundancy } & & \end{array}$




\section{"She worked hard yet without complaining."}

The conjunction "yet" cannot be used to coordinate a verb phrase and a prepositional phrase in this sentence. Besides, the sentence is complete without this conjunction.

\section{Example 8 Subordinating Conjunction Redundancy \\ "Most of the students who studying abroad did not want to come back."}

The students mingled the attributive sentence introduced by the antecedent "who" with the postpositive attributive marked by the present participle form. Since the noun "students" is modified by a postpone attributive with the same function of an attributive clause, the subordinating conjunction is seen as redundant.

\section{Example 9 Preposition Redundancy}

"Some students will even quit the jobs they just find and to seek another."

The conjunctions are used to connect words sharing the same part of speech or phrases with the same structure. Therefore, the preposition "to" is supposed to be deleted from this sentence which is determined by the usage of conjunctions "and" since it cannot coordinate a verb phrase and a prepositional phrase.

\section{Example 10 Article Redundancy}

"If you have courage of facing the all kinds of the difficulties, studying abroad is a good opportunity for you."

The definite article in English is used to qualify the noun that follows the article as a particular thing to which the speaker is referring. However, the noun is followed by another modifier "all kinds of" without indicating some difficulties whose meaning contradicts with the definite article which is supposed to be removed from this sentence.

\section{Example 11 Auxiliary Verb Redundancy}

"Many people are regret that they didn't get degrees when they were students."

The sentence meaning is expressed in the active voice in the present simple tense, so the auxiliary verb violates the grammar rule that only the progressive tense or passive voice is rendered by different forms of auxiliary "be".

From the above examples extracted from CLEC, it can be found that the redundancy errors within sentences can all be attributed to the lexicons, which serve as the basic unit of language. Specifically, message repetition or entailment, in the form of semantic redundancy errors, can be attributed to the misuse of content words including nouns, verbs, pronouns, adjectives and adverbs. Except for verb redundancy, all the errors from other parts of speech tend to emerge as a modifier in phrases whose meaning has been partially or totally implied by the word they modify. Meanwhile, syntactic redundancy errors mainly result from the overuse of functional words, such as conjunctions, prepositions, articles, and auxiliary verbs. Since functional words have no actual meaning, they merely violate the grammar rules without indicating message repetition or entailment.

\subsection{Redundancy errors for students with different language proficiency}

Designed on the basis of the results from the corpus-based study, the questionnaire for the annotation test consists of fourteen questions each with one redundancy error from different parts of speech. The first seven questions containing semantic redundancy errors result from a redundant content word, namely adverb as the modifier of a verb, adverb as the modifier of an adjective, adjective, noun, verb and pronoun. The syntactic redundancy errors in the other seven questions are caused by a redundant function word, such as article, preposition, conjunction and auxiliary verb. The results from the annotation test are displayed in the following tables.

Table 1. The error rates for semantic redundancy errors

\begin{tabular}{|c|c|c|c|}
\hline $\begin{array}{l}\text { Semantic } \\
\text { Redundancy } \\
\text { errors }\end{array}$ & Subsets & $\begin{array}{l}\text { Upper } \\
\text { level }\end{array}$ & $\begin{array}{l}\text { Lower } \\
\text { level }\end{array}$ \\
\hline 1. compete together & $\begin{array}{l}\text { Adverb } \\
\text { (modifier of } \\
\text { V.) }\end{array}$ & $16.67 \%$ & $36.67 \%$ \\
\hline 2. is booming fast & $\begin{array}{l}\text { Adverb } \\
\text { (modifier of } \\
\text { Adj.) }\end{array}$ & $40 \%$ & $46.67 \%$ \\
\hline 3. be relatively better & $\begin{array}{l}\text { Adverb } \\
\text { (modifier of } \\
\text { Adj.) }\end{array}$ & $60 \%$ & $66.67 \%$ \\
\hline 4. is full of so much & Adjective & $10 \%$ & $16.67 \%$ \\
\hline 5. secretary job & Noun & $13.33 \%$ & $33.33 \%$ \\
\hline $\begin{array}{l}\text { 6. There } \\
\text { are ... remain... }\end{array}$ & Verb & $46.67 \%$ & $63.33 \%$ \\
\hline
\end{tabular}




\section{7. their...themselves Pronoun $\quad 33.33 \% \quad 50 \%$}

Table 1 compares the error rates of annotating semantic redundancy errors for the students with high language proficiency and those with low language proficiency while Table 2 displays the error rates of identifying the syntactic redundancy errors for the two groups in the annotation test. In general, the upper-level group achieve a better performance when annotating the redundancy errors in both two categories compared with the lower-level group.

According to Table 1, the error rates appear to be higher for the lower-level group in all the 7 questions containing message repetition or entailment marked by content words. The reoccurrence of adjectives or their synonyms can be easily identified in the annotation test as the overwhelming majority of the experimental subjects in both groups can annotate this subset of redundancy errors. By contrast, students appear to have the worst performance when annotating redundant adverbs functioning as modifiers of adjectives. The error rates in questions containing redundant adverbs are shown to be the highest with up to $60 \%$ for the upper-level group and $66.67 \%$ for the lower-level group, which means over half of the participants cannot identify the redundant adverbs without realizing the message entailment in adverb-adjective phrases. Obviously, college students have mastered the phrasal structure of emphasizing the degree of adjectives by adding an adverb in the front but do not understand whether there is semantic overlap in adverb-adjective phrases where the meaning of adverbs has been contained in the word they modify. Having the second highest error rates at $46.67 \%$ and $63.33 \%$ respectively, redundant verbs are also hard for them to annotate, which indicates students can hardly detect the message repetition of verbs in existential sentences. For the upper-level group, the error rates of annotating redundant nouns, adverbs as a modifier of adjectives are relatively low with below 20 percent. These two subsets of redundancy errors are also less common for their counterparts. However, it seems to be hard for the lower-level group to annotate the redundant pronouns referring to the same nouns since the error rate for this subset reaches $50 \%$.

Table 2. The error rates for syntactic redundancy errors

\begin{tabular}{llll}
\hline $\begin{array}{l}\text { Syntactic } \\
\text { redundancy } \\
\text { errors }\end{array}$ & Subsets & $\begin{array}{l}\text { Upper } \\
\text { level }\end{array}$ & $\begin{array}{l}\text { Lower } \\
\text { level }\end{array}$ \\
\hline 1. all kinds of the & article & $13.33 \%$ & $33.33 \%$ \\
2. the science & article & $23.33 \%$ & $36.67 \%$
\end{tabular}

$\begin{array}{llll}\begin{array}{l}\text { 3. enter into the } \\ \text { room }\end{array} & \text { preposition } & 23.33 \% & 46.67 \% \\ \text { 4. go to upstairs } & \text { preposition } & 30 \% & 36.67 \% \\ \text { 5. who } & \text { conjunction } & 53.33 \% & 63.33 \% \\ \text { 6. yet } & \text { conjunction } & 26.67 \% & 36.67 \% \\ \text { 7. are regret } & \begin{array}{l}\text { auxiliary } \\ \text { verb }\end{array} & 46.67 \% & 66.67 \% \\ \end{array}$

As shown in Table 2, the accuracy for students with higher English proficiency outnumbers that for their counterparts again in the questions containing syntactic redundancy errors. The difference values for annotating redundant functional words ranging from $6.67 \%$ to $23.34 \%$ are a bit larger. The overuse of subordinating conjunctions in complex sentences and the improper addition of auxiliary verbs in predicates are of most prominence since even almost $50 \%$ of the upper-level group cannot annotate these two subsets of redundancy errors. These figures uncover a fact that college students have difficulty in mastering the sentence structure, especially that of complex sentences, which proves that Chinese English learners tend to make syntactic redundancy errors in the sentence level. It is also noticeable that both the largest and smallest difference values between the two groups emerge in test questions containing redundant prepositions. The striking contrast reveals that the accuracy for students' usage of prepositions is determined by their mastery with fixed collocations, and the improper use of prepositions tends to decrease dramatically with an increasing familiarity for fixed collocations.

\section{DISCUSSION}

\subsection{Reasons for Redundancy}

This research analyzes the causes for redundancy errors from two aspects under the framework of error analysis. Specially, language transfer accounts for interlanguage errors shown as superfluous content words while language proficiency explains intralingual errors caused by the overuse of functional words.

\subsubsection{Negative transfer}

During the process of learning a second language, the influence of L1 thinking is evident. Pinker supported that language was influenced by thinking patterns [17]. He claimed that "the way language works is that each person's brain contains a lexical of words and the concepts they stand for (a mental dictionary) and a set of rules that combine the words to convey relationships among concepts (a mental grammar)" [17]. Since 
Chinese and English belong to Indo-European and Sino-Tibetan language families respectively, there exists a significant language distance which refers to an obvious distinction between the two languages [18] [19]. As the result, the influence of L1 thinking on English learners is mainly manifested as the negative transfer.

The redundancy errors as content words are mainly caused by superfluous modifiers whose meanings are partly or entirely entailed in the words they modify. At the phrase level, students tend to use adjectives and nouns to express the attribute or scope of a particular object. Also, they add information about the action or situation by inserting adverbs in front of verbs or adjectives. Redundancy errors in this sense are correct syntactically but incorrect semantically since Chinese English learners are unaware of the message repetition or entailment conveyed by words or phrases in English. One possible explanation for this category of redundancy errors is the influence of native thinking since L2 learners tend to do literal translation without realizing the semantic gap or overlap between the source language and target language.

As for semantic redundancy errors in sentence level, they are mainly caused by superfluous verbs whose original purpose is to provide supplementary information for predicates to denote an action or a state. The serial verb construction is unique and common in Chinese, but they are seen as syntactically incorrect from literal translation since they violate the English sentence structure. This is because the juxtaposition of bare verb phrases (VP) are not allowed in languages whose morphology is rich including English, since the overt relationship between VPs must be encoded explicitly. However, the encoding of structural relations isn't necessary for languages such as Chinese with poor morphology, and the presence of grammatical markers is optional in syntax [20].

It is important to realize that even though some similarities exist between Chinese and English, there are many differences between syntactic and lexical knowledge of the two languages, thereby redundancy errors, as an integral part of improper collocations, are common in L2 learners' language performance due to the negative transfer of their L1. In this way, students' use of L1 thinking in word choice affects the accuracy of collocations in a second language. Also, sentence structures in their native language are likely to be transferred in English writing tasks. The sentence formation and word choice for L2 learners are likely to be shaped by their native language with part of syntactically and semantically differentiated knowledge.

\subsubsection{Language proficiency}

Functional words, such as a preposition, auxiliary verb and conjunction, express primarily a grammatical relationship. Syntactic redundancy errors shown as redundant functional words can be attributed to language learners' inadequate knowledge of grammar rules. Translated into Chinese, there are no articles or prepositions as equivalents of those in English, which proves these redundant functional words are not a result of their L1 [21]. Therefore, the syntactic category of redundancy errors is seen as intralingual errors since they are found within the structure of English itself, and through reference to the strategy by which a second language is acquired and taught [22].

The overuse of "to" is a typical example of intralingual errors. Grammar rules of English define those prepositions that are used to combine with a noun or pronoun or noun phrase to form a prepositional phrase and they are restricted as necessary constituents between intransitive verbs and nouns. However, students are found to use "to" after transitive verbs or in front of adverbs in such writing samples as "discuss about the plan" and "go to upstairs". The overuse of the auxiliary verb "be" also reveals L2 learner's lack of knowledge that it cannot be linked to the base form of a verb to serve as the predicate together in a sentence.

As for intralingual redundancy errors, L2 learners could significantly reduce redundant functional words with a better grasp of grammar rules in English.

\subsection{Pedagogical Implications}

In this paper, several recommendations are presented to make pedagogical suggestions for English instructors and learners in China to reduce redundancy errors and ensure the conciseness and accuracy of English writing.

Firstly, instructors should be aware of the interference of learners' native language. Understanding the influence of L1 thinking on Chinese students' second language acquisition can help educators to have clearer thoughts when sorting out educational plans and strategies and enable both teachers and students to realize the importance of cultivating students' second language thinking.

Secondly, the occurrence of errors should not be seen as a failure in acquiring a second language, it should be regarded as a significant sign of the learners' second language development instead. Redundancy errors can be used to check whether students have adequate knowledge of grammar rules. English teachers can identify students' stages of language development based on the redundancy errors they make during the writing process so that they can emphasize the error-prone collocations and sentence structures accordingly. 


\subsection{Research Contributions}

The correct collocation in productive tasks is an important criterion to measure the foreign language proficiency of a second language learner truly and comprehensively. However, the emergence of redundancy errors, as a part of improper wrong collocations, can severely damage the message transmission in daily communication and formal writing. The research on redundancy errors and the possible causes for them is of practical value to know which category or subsets of redundancy errors are frequent in productive writing tasks and hard for students to annotate.

Since this research provides a comprehensive demonstration of redundancy errors in college students' writing samples, it finds out the redundancy errors from CLEC in different linguistic levels can all be attributed to the lexicon which serves as the basic unit of language. This research innovates the classification of redundancy errors by grouping them into two main categories in terms of semantics and syntax with a further division of ten subsets as shown in different parts of speech, namely nouns, pronouns, verbs, adjectives, adverbs, articles, prepositions, conjunctions and auxiliary verbs.

The theoretical model has also been innovated by combining two tasks for error analysis to test students' language ability in a productive task and a receptive task. Most of the previous studies adopt discourse analysis to calculate the most frequent redundancy errors under the framework of error analysis which continues as a useful procedure for the study of SLA [23]. However, error analysis also has its drawback since focusing on errors alone does not necessarily provide information on what the L2 learner has acquired [16]. The occurrence of some redundancy errors may not serve as a typical and common error for learners with the same language proficiency. This research designs an annotation test as the supplement for the discourse analysis to fill in this gap. After collecting and analyzing the redundancy errors in productive tasks, college students are asked to attend an annotation test to see whether these redundancy errors are occasional or common among them.

\subsection{Limitations}

In this paper, there are two limitations of this research.

Primarily, the corpus-based collection is not comprehensive enough since there are five subsets in CLEC amounting to more than one million words of written samples. As the classification for redundancy errors in this research includes two main categories shown as semantic redundancy errors resulting from the superfluous content words and syntactic redundancy caused by the overuse of functional words, the researcher only selects redundancy errors of each part of speech from this corpus but not compare the distribution of redundancy errors among four subsets in this corpus.

Additionally, the annotation results show the statistical limitations of the study. The questionnaire in the annotation test consists of fourteen choice questions, which means there are only one or two questions about redundancy errors from each part of speech. The sentences with redundancy errors maybe not typical enough as the test questions. More test questions are expected to be added to the annotation test.

\section{CONCLUSION}

This research paper investigates the common redundancy errors for non-English majors in China and explores their relationships with language proficiency. This research adopts the CLEC to conduct discourse analysis. The four subsets of college students' writing samples are selected from the corpus to collect redundancy errors as different parts of speech in phrasal level and sentence level. The analysis shows that superfluous content words result in semantic redundancy errors while the overuse of functional words leads to syntactic redundancy errors. The corpus-based study is supplemented with an annotation test to test which category or subsets of redundancy errors are difficult for English learners with different proficiency. The results reveal that the redundant adverbs, verbs, auxiliary verbs and conjunctions are most difficult for college students although the upper-level group has a better language performance. Under the framework of error analysis, this research provides reasonable explanations for the occurrence of redundancy errors, such as negative transfer and inadequate knowledge of the target language. This research also provides some pedagogical implications for English instructors and students to reduce redundancy errors in English writing tasks. Although there are still some limitations including the incomplete collection of redundancy errors from the corpus and a limited number of test questions in the investigation, it provides new insights into the study on redundancy errors by decomposing them into different parts of speech and by combing discourse analysis and corpus linguistics together.

\section{REFERENCES}

[1] C.E. Shannon, W. Weaver, The Mathematical Theory of Communication, Urbana: The University of Illinois Press, 1949.

[2] F.X. Wang, English-Chinese Comparative Semantics, Beijing: Foreign Language Press, 2001. 
[3] A.M. Rosie, Information and Communication Theory, London: Van Nostrand Reinhold Company, 1973.

[4] F. Reza, Information Theory, Beijing: Foreign Language Press, 1990.

[5] S.H. Xu, The redundancy of language, Modern Foreign Languages (2) (1984) 3-8. DOI: CNKI:SUN:XDWY.0.1984-02-000.

[6] Wit, E. C., \& Gillette, M. (1999). What is Linguistic Redundancy? Technical Report. Retrieved from https://citeseerx.ist.psu.edu/viewdoc/download?doi $=10.1 \cdot 1.714 .5763 \&$ rep $=$ rep $1 \&$ type $=$ pdf

[7] Z.H. Shao, Study and Contrast on English and Chinese, East China University of Science and Technology, 1997.

[8] Y.Z. Liu, Y.H. Xiang, A Study on Redundancy in English Majors' Compositions, Journal of Educational Institute of Jilin Province 34(9) (2018) 71-74.

Doi: 10.16083/j.cnki.1671-1580.2018.09.020.

[9] J.Q. Li, J.J. Cai, The Misuse of the English Articles in Compositions of Chinese College Students-A Corpus-Based Study, Journal of PLA University of Foreign Language (6) (2001) 58-62. Doi: CNKI:SUN:JFJW.0.2001-06-013.

[10] X.M. Liu, S.L. Liu, A Corpus-Based Investigation of Two Types of Errors: Absence and Redundancy Committed by Chinese EFL Learners, Technology Enhanced Foreign Languages (3) (2004) 74-76. Doi:CNKI:SUN:WYDH.0.2004-03-019.

[11] X.M. Liu, Absence and Redundancy of and Committed by Chinese EFL Learners--A Corpus-Based Research, Technology Enhanced Foreign Languages (2) (2005) 44-46. Doi:CNKI:SUN:WYDH.0.2005-02-010.

[12] J.S. Song, Absence and Redundancy of so Committed by Chinese EFL Learner, Journal of Jiangsu University of Science and Technology (Social Science Edition) 7(2) (2007) 95-98. Doi:10.16148/j.cnki.cn32-1743/c.2007.02.022.
[13] L.L. Tang, Error Analysis on the Absence and Redundancy of BUT Committed by Chinese EFL Learners--A Corpus-Based Research, Journal of Liaoning Economic Management Cadre College (3) (2010) 109-110. Doi:CNKI:SUN:LNJJ.0.2010-03-056.

[14] C.L. Jiang, A Study on Redundancy Errors in College English Writing, Journal of Lanzhou Institute of Education (1) (2012) 68-70. Doi:CNKI:SUN:LNJJ.0.2010-03-056.

[15] L. Cai, The Transfer Redundancy in Chinese English Learners' Writing, Journal of Changchun Institute of Technology (Social Science Edition) 19(2) 76-80. DOI:CNKI:SUN:CGCS.0.2018-02-018.

[16] M. Saville-Troike, Introducing Second Language Acquisition, Cambridge University Press, 2014

[17] S. Pinker, The Language Instinct: How the Mind Creates Language, Harper Perennial Modern Classics, 1994.

[18] E. Kellerman, Towards a Characterisation of the Strategy of Transfer in Second Language Learning, Interlanguage Studies Bulletin 2(1) (1977) $58-$ 145. DOI: https://www.jstor.org/stable/43135159

[19] H. Ringbom, The Influence of the Mother Tongue on the Translation of Lexical Items, Interlanguage Studies Bulletin 3(1) (1978) 80-101. DOI: https://www.jstor.org/stable/43135191

[20] X.D. Zheng, The Tense of Serial Verb Constructions, Modern Foreign Languages(Bimonthly) 41(4) (2018) 453-466.

[21] Y. N. Liu, 2008. A Corpus-Based Analysis of Redundancy Errors in English Writings by Chinese EFL Learners. Northeast Normal University.

[22] J.C. Richards, Error Analysis: Perspectives on Second Language Acquisition, London: Longman Group Limited, 1978.

[23] S.P. Corder, The Significance of Learners Language Errors, International Review of Applied Linguistics (5) (1967) 161-170. 